\title{
A survey of zoonotic pathogens carried by Norway rats in Baltimore, Maryland, USA
}

\author{
J. D. EASTERBROOK ${ }^{1 *}$, J. B. KAPLAN ${ }^{1}$, N. B. VANASCO ${ }^{3}$, W. K. REEVES ${ }^{4}$ \\ R. H. PURCELL ${ }^{5}$, M. Y. KOSOY ${ }^{6}$, G. E. GLASS ${ }^{1}$, J. WATSON$^{2}$ AND S. L. KLEIN ${ }^{1}$ \\ ${ }^{1}$ The W. Harry Feinstone Department of Microbiology and Immunology, The Johns Hopkins Bloomberg School \\ of Public Health, Baltimore, MD, USA \\ ${ }^{2}$ Department of Molecular and Comparative Pathobiology, The Johns Hopkins University School of Medicine, \\ Baltimore, MD, USA \\ ${ }^{3}$ Instituto Nacional de Enfermedades Respiratorias (INER) 'E. Coni', Administración Nacional de Laboratorios e \\ Institutos de Salud (ANLIS), Blas Parera 8260, Santa Fe, Argentina \\ ${ }^{4}$ Centers for Disease Control and Prevention, Atlanta, GA, USA \\ ${ }^{5}$ Hepatitis Viruses Section, Laboratory of Infectious Diseases, National Institute of Allergy and Infectious \\ Diseases, National Institutes of Health, Bethesda, MD, USA \\ ${ }^{6}$ Division of Vector-Borne Infectious Diseases, National Center for Infectious Diseases, Centers for Disease \\ Control and Prevention, Fort Collins, CO, USA
}

(Accepted 9 November 2006; first published online 15 January 2007)

\section{SUMMARY}

Norway rats (Rattus norvegicus) carry several zoonotic pathogens and because rats and humans live in close proximity in urban environments, there exists potential for transmission. To identify zoonotic agents carried by rats in Baltimore, Maryland, USA, we live-trapped 201 rats during 2005-2006 and screened them for a panel of viruses, bacteria, and parasites. Antibodies against Seoul virus (57.7\%), hepatitis E virus (HEV, 73.5\%), Leptospira interrogans $(65.3 \%)$, Bartonella elizabethae (34.1\%), and Rickettsia typhi (7.0\%) were detected in Norway rats. Endoparasites, including Calodium hepatica (87.9\%) and Hymenolepis sp. (34.4\%), and ectoparasites (13.9\%, primarily Laelaps echidninus) also were present. The risk of human exposure to these pathogens is a significant public health concern. Because these pathogens cause non-specific and often self-limiting symptoms in humans, infection in human populations is probably underdiagnosed.

\section{INTRODUCTION}

Norway rats (Rattus norvegicus) are prevalent in urban environments and pose a threat to public health, both through their destructive behaviour and by serving as reservoirs for pathogens that can be transmitted to humans. A survey of residents of

\footnotetext{
* Author for correspondence: J. D. Easterbrook, The W. Harry Feinstone Department of Microbiology and Immunology, The Johns Hopkins Bloomberg School of Public Health, 615 N. Wolfe Street, Baltimore, MD 21205, USA.

(Email: jeasterb@jhsph.edu)
}

Baltimore, Maryland found that nearly two-thirds of respondents $(64 \%)$ observed rats in streets and alleys, $6 \%$ saw rats inside residences, and $1 \cdot 2 \%$ had experienced a rodent bite in their lifetime [1]. Although Norway rats are reported to be hosts for a large number of pathogens [2], a comprehensive survey of pathogens carried by rats in an urban setting has not been conducted. In urban environments, humans and rats live in close proximity and the potential for spillover of zoonotic agents poses a public health concern that has rarely been evaluated. To identify and assess the prevalence of zoonotic agents carried by 
rats in an urban environment, we conducted a survey of pathogens carried by Norway rats in Baltimore, Maryland in 2005-2006, including assessment of the prevalence of Seoul virus, hepatitis E virus (HEV), lymphocytic choriomeningitis virus (LCMV), Leptospira interrogans, Bartonella elizabethae, and Rickettsia typhi by serological analyses, and the presence of Hymenolepis sp. and Calodium (syn. Capillaria) hepatica.

\section{METHODS}

\section{Wild-caught rats}

Adult male and female $R$. norvegicus were livetrapped (Tomahawk Trap Co., Tomahawk, WI, USA) from 20 locations in neighbourhoods in East Baltimore, Maryland. Rats were trapped from April 2005 to April 2006. The sampling strategy was designed to trap similar numbers of rats in each season to account for possible seasonal variation in pathogen prevalence. All trapping locations were in urban areas in alleys behind residential dwellings. Traps were baited with peanut butter and set at locations $\sim 1-2 \mathrm{~h}$ before sundown. Details of sampling procedures have been previously described [3]. Rats were collected and processed the next morning. Rats were euthanized using $\mathrm{CO}_{2}$, weighed, sexed, and bled by cardiac puncture. Serum was stored at $-80{ }^{\circ} \mathrm{C}$ until serological analysis. Each rat was examined for ectoparasites using a fine comb. Faecal and caecum content samples were collected for helminth ova analysis. The Johns Hopkins Animal Care and Use Committee (protocol no. RA05H6) approved all procedures described in this study.

\section{Serological analyses}

\section{Seoul virus}

Anti-Seoul virus IgG was measured by ELISA as previously described [4]. Microtitre plates were coated with lysate from Vero E6 cells infected with Seoul virus or from uninfected Vero E6 cells. Sera from experimental and control rats were diluted $1: 100$ and added to plates in duplicate. Secondary antibody [alkaline phosphatase-conjugated anti-rat IgG; Kirkegaard and Perry Laboratories (KPL), Gaithersburg, MD, USA] was added and developed with $p$-nitrophenylphosphate substrate buffer. Optical density (OD) was measured at $405 \mathrm{~nm}$ and the average OD for each set of uninfected Vero E6 duplicates was subtracted from the average OD for each set of infected Vero E6 duplicates. Samples were considered positive if the average adjusted OD was $\geqslant 0 \cdot 100 \mathrm{~nm}$.

\section{HEV}

Anti-HEV IgG was measured by ELISA as previously described [5, 6]. Microtitre plates were coated with ORF2 antigen $(0 \cdot 1 \mu \mathrm{g} /$ well $)$. After blocking, sera from test and control rats were diluted 1:100 and added to plates in duplicate. Secondary antibody [horseradish peroxidase (HRP)-labelled goat anti-rat IgG (KPL)] was added and developed with azino-diethylbenzotyazol-sulfonate (ABTS) substrate. OD at $405 \mathrm{~nm}$ was measured and the cut-off was established for each test from internal controls; throughout this study the cut-off OD averaged $0 \cdot 370$.

\section{$L C M V$}

Serology samples were submitted to a commercial laboratory (BioReliance SM, Rockville, MD, USA). Antigen from LCMV strain CA1371 (obtained from Wallace P. Rowe) grown in Vero E6 cells was used for the ELISA assays. Tests positive by ELISA were confirmed by IFA.

\section{Leptospira sp.}

Anti-L. interrogans IgG was measured by ELISA as previously described [7]. Microtitre plates were coated with sonicated antigen prepared from cultures of Leptospira serovars Tarassovi and Pyrogenes $(0 \cdot 1 \mu \mathrm{g} /$ well). Sera from test and control rats were diluted $1: 100$ and added to plates in duplicate. Secondary antibody (peroxide-conjugated anti-rat IgG (Sigma, St Louis, MO, USA) was added and developed with tetramethylbenzidine (TMB). Following termination of the enzyme-substrate reaction with $\mathrm{H}_{2} \mathrm{SO}_{4}$, the OD was measured at $450 \mathrm{~nm}$. The OD was standardized by dividing the sample OD by the OD of the pooled negative controls and samples were considered positive when the standardized OD was $>2 \cdot 4$. Leptospira serogroups were identified by a microagglutination test (MAT) with 10 serotypes of $L$. interrogans as previously described [7]. The end-point titre was determined as the highest serum dilution (minimum $1: 20$ ) showing agglutination of at least $50 \%$ of the cells.

\section{Rickettsia typhi}

Anti-Rickettsia sp. IgG was measured by IFA as previously described [8]. R. typhi (Wilmington strain) 
grown in DH-82 cells were dotted onto slides. Sera from test and control rats were diluted to $1: 32$ and added to slides. Secondary antibody [FITC conjugated goat anti-rat IgG (KPL)] was added and slides were mounted with a glass coverslip over a glycerolbased mounting medium. Sera were determined to be positive when discrete, fluorescent organisms were visible. Sera that were positive at $1: 32$ were retested at $1: 64$ and $1: 100$. To determine cross-reactivity, slides were dotted with $R$. akari (Kaplan strain) grown in egg yolk sac. Samples with positive IFA titres to $R$. typhi were tested against $R$. akari at a 1:64 dilution. For all antigens, a positive serum was defined as a titre of $\geqslant 1: 64$.

\section{Bartonella elizabethae}

Anti-B. elizabethae IgG was measured by IFA. $B$. elizabethae bacteria (strain F9251) grown in Vero E6 cells were dotted onto poly-L-lysine-coated slides, air dried, and fixed in $1 \%$ paraformaldehyde for $1 \mathrm{~h}$. Plates were washed with PBS (three times for $5 \mathrm{~min}$ ) in between each step. Following blocking with PBS $+10 \%$ FBS, sera from test and control samples were diluted 1:50 in PBS $+2 \%$ FBS and $15 \mu 1$ dotted on the appropriate well. Slides were incubated for 30 min at $37^{\circ} \mathrm{C}$ and secondary antibody [FITC conjugated goat anti-rat $\operatorname{IgG}(\mathrm{H}+\mathrm{L}, \mathrm{KPL})]$ was diluted 1:100 in PBS and added to each well. Slides were incubated in the dark for $30 \mathrm{~min}$ at $37{ }^{\circ} \mathrm{C}$, dried, and mounted with a coverslip after adding a small drop of glycerol to each well. Sera were determined to be positive when discrete, fluorescent organisms were visible.

\section{Calodium hepatica}

C. hepatica adults and eggs were visible as yellowishwhite lesions in rat livers and a subset of adults were verified by light microscopy $(100 \times$ magnification $)$.

Hymenolepis sp. faecal and caecum content floats

Faecal and caecum content samples were homogenized in zinc sulphate buffer $(400 \mathrm{~g} / \mathrm{l})$ in glass test tubes and filled to the brim with buffer. A coverslip was placed on top for $15 \mathrm{~min}$ and transferred to a slide for microscopic evaluation. Both H. nana and $H$. diminuta ova were identified, but were not differentiated in data records. Helminth ova identification was conducted after initiation of this study; thus fewer rats were examined for helminth infection compared with serological analyses.

\section{Statistics}

Differences in pathogen prevalence by various demographic strata included age, sex, seasonality, and pregnancy status and were evaluated by $\chi^{2}$ or Fisher's exact tests. Weight was used as a correlate of age as follows: juveniles were $<200 \mathrm{~g}(n=31)$, young adults were $200-399 \mathrm{~g}(n=71)$, and adults were $\geqslant 400 \mathrm{~g}$ $(n=98)$. Rats over $200 \mathrm{~g}$ were sexually mature as indicated by the decent of testes in males and development of vaginal openings in females. Seasons were defined as: winter (December-February), spring (March-May), summer (June-August), and autumn (September-November). Correlational analyses were conducted using Pearson product moment. Comparisons were considered statistically significant at $P<0 \cdot 05$.

\section{RESULTS}

\section{Prevalence of zoonotic pathogens}

Prevalence of antibody or rodent-borne pathogens is presented in decreasing order (Table). The most common pathogen was the nematode $C$. hepatica (87.9\%, 176/201). Antibodies against HEV (73.5\%, $144 / 196)$ and Seoul virus $(57 \cdot 7 \%, 116 / 201)$, as well as L. interrogans $(65 \cdot 3 \%, 124 / 190)$, were detected in over half of the Norway rats tested. The tapeworms $H$. nana or $H$. diminuta were observed in more than one-third of rats $(34.0 \%, 55 / 162)$. Seroprevalence for ectoparasite-borne bacteria was highest for B. elizabethae $(34 \cdot 1 \%, 63 / 197)$, followed by $R$. typhi $(7 \cdot 0 \%, 14 / 201)$. Antibodies against LCMV were not detected in a subset of rats that were tested $(0 / 48)$.

The spiny rat mite (Laelaps echidninus) was the most prevalent ectoparasite $(12 \cdot 4 \%, 25 / 201)$. Two cat fleas (Ctenocephalides felis) and three tropical rat mites (Ornithonyssus bacoti) also were collected during the summer months. A representative selection of serum samples that tested positive for L. interrogans $(n=15)$ were tested by MAT and showed specific titres against L. copenhageni (Icterohaemorrhagiae serogroup).

\section{Body size}

The presence of antibodies against Seoul virus, HEV, and $L$. interrogans significantly increased with age class $\left(\chi^{2}=53 \cdot 67,2, P<0 \cdot 001 ; \chi^{2}=57 \cdot 25,2, P<0 \cdot 001\right.$; $\chi^{2}=48 \cdot 06,2, P<0 \cdot 001$, respectively) (Fig). Prevalence of $C$. hepatica was significantly higher in young adults 
Table. Prevalence of zoonotic pathogens in Norway rats from Baltimore, Maryland, USA 2005-2006

\begin{tabular}{llll}
\hline \hline Zoonotic pathogen & Prevalence & $\begin{array}{l}\text { Number of } \\
\text { rats positive }\end{array}$ & $\begin{array}{l}\text { Total no. } \\
\text { of rats* }\end{array}$ \\
\hline Calodium hepatica $\dagger$ & $87 \cdot 9$ & 176 & 201 \\
Hepatitis E virus $\$$ & $73 \cdot 5$ & 144 & 196 \\
Leptospira interrogans $\ddagger$ & $65 \cdot 3$ & 124 & 190 \\
Seoul virus & $57 \cdot 7$ & 116 & 201 \\
Bartonella elizabethae§ & $34 \cdot 1$ & 63 & 197 \\
Hymenolepis sp. $\dagger$ & $34 \cdot 0$ & 55 & 162 \\
Rickettsia typhi§ & $7 \cdot 0$ & 14 & 48 \\
Lymphocytic choriomeningitis & 0 & 0 & \\
virus $\$$ & & & \\
\hline \hline
\end{tabular}

* Unequal sample sizes due to sample availability.

$\uparrow$ Pathogen prevalence determined by microscope evaluation.

+ Seroprevalence determined by ELISA.

$\S$ Seroprevalence determined by IFA.

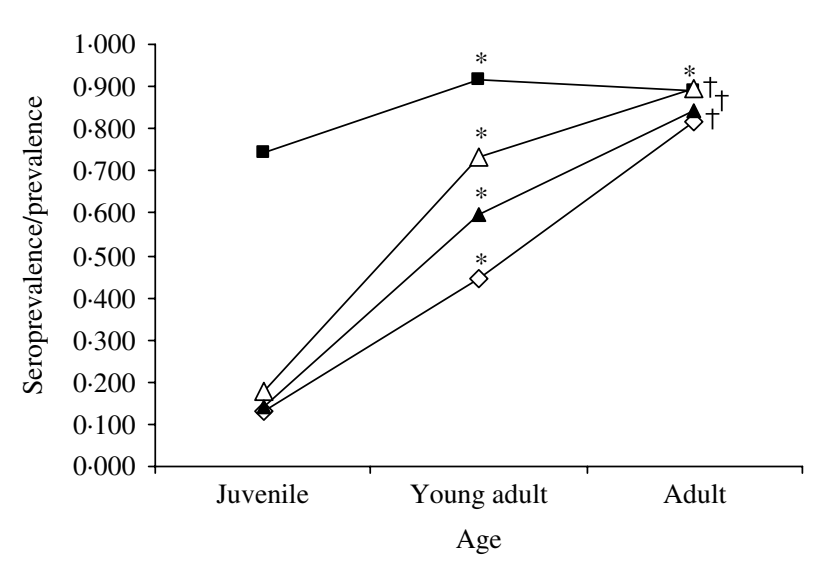

Fig. Seroprevalence of Seoul virus $\left(-\diamond_{-}\right)$, HEV $\left(-\triangle^{-}\right)$, and L. interrogans $\left(-\boldsymbol{\Delta}_{-}\right)$, as well as prevalence of $C$. hepatica $(-\boldsymbol{-})$ for three age groups of rats: juveniles $(<200 \mathrm{~g})$, young adults $(200-399 \mathrm{~g})$, and adults $(\geqslant 400 \mathrm{~g})$. * Seroprevalence young adult and adult $>$ juvenile and $\dagger$ seroprevalence adult $>$ young adult and juvenile, $P<0 \cdot 05$.

and adults compared with juveniles $\left(\chi^{2}=6 \cdot 33,2\right.$, $P=0.042$ ) (Fig.). Seroprevalence of $B$. elizabethae and $R$. typhi as well as the prevalence of ectoparasites and Hymenolepis sp. did not differ according to age.

\section{Sex}

Comparable numbers of males $(n=105)$ and females $(n=96)$ were collected and there was no difference in the numbers of males and females trapped by age class $(P>0 \cdot 05)$. The prevalence of Hymenolepis sp. was higher in males compared with females $\left(\chi^{2}=\right.$ $6 \cdot 46,1, P=0.011)$. Conversely, the prevalence of antibodies against $L$. interrogans was higher in females compared with males $\left(\chi^{2}=4 \cdot 52,1, P=0 \cdot 033\right)$. Sex differences were not observed in association with antibodies against Seoul virus, HEV, B. elizabethae, or $R$. typhi, or the presence of $C$. hepatica or ectoparasites $(P>0 \cdot 05$ for all $)$.

\section{Seasons}

Attempts were made to collect similar numbers of rats during each season, but autumn was especially rainy, so trapping success was reduced during this time period $(n=20)$. Numbers of rats trapped during other seasons, i.e. winter $(n=64)$, spring $(n=54)$, and summer $(n=63)$ were otherwise comparable. Seasonal differences were observed for Hymenolepis sp.: the prevalence was significantly lower in spring $(17 \cdot 4 \%)$ compared with summer $(45 \cdot 5 \%)$, autumn $(40 \cdot 0 \%)$ and winter $(38.1 \%)\left(\chi^{2}=8 \cdot 38,3, P=0 \cdot 04\right)$. The prevalence of spiny rat mites was significantly higher in summer and autumn $(23.8 \%$ and $25 \%$, respectively) compared with winter and spring $(4 \cdot 7 \%$ and $3 \cdot 7 \%$, respectively, $\left.\chi^{2}=21 \cdot 08,3, P<0 \cdot 001\right)$. There were no significant seasonal patterns in the prevalence of Seoul virus, HEV, L. interrogans, B. elizabethae, R. typhi, and C. hepatica observed.

\section{Pregnancy}

Thirty-three percent $(32 / 96)$ of the female rats were pregnant at the time of trapping, with the highest rate of pregnancy during winter $(52.0 \%)$ and the lowest during summer $(16 \cdot 1 \%)$. Pregnant females were 
significantly more likely to have antibody against Seoul virus than were non-pregnant females $\left(\chi^{2}=\right.$ $3 \cdot 94,1, P=0 \cdot 047)$. Age was a confounding factor and after stratification by age class, the effect of pregnancy on infection no longer exists among adult females (for young adults, Fisher's exact test, $P>0.05$ and for adults, $\chi^{2}=1 \cdot 28, P>0 \cdot 05$ ). There was no effect of pregnancy on the presence of antibodies against HEV, L. interrogans, B. elizabethae, or R. typhi, or the presence of $C$. hepatica, Hymenolepis sp., or ectoparasites $(P>0 \cdot 05$ for all).

\section{Correlations}

There was a correlation between prevalence of $L$. interrogans and HEV $(r=0 \cdot 36, P<0 \cdot 001)$. No significant correlation existed between Seoul virus infection and $L$. interrogans or HEV infection $(r<0 \cdot 1$, $P>0.05$ ); in fact, the presence of antibodies against Seoul virus was not correlated with the likelihood of being infected with any of the other pathogens tested $(P>0.05$ for all tests).

\section{DISCUSSION}

Norway rats serve as reservoirs for a variety of zoonotic pathogens. The panel of pathogens was selected because these organisms have been identified in both humans and rats in urban environments and resources were readily available for testing in rats. Increasing age-related seroprevalence of Seoul virus, HEV, L. interrogans, and C. hepatica in rats has been previously documented and probably reflects an increased probability of encountering pathogens with age $[4,9-11]$. The impact of pregnancy on infection has not been reported in wild rat populations and seems to have little effect on seroprevalence of viruses and bacteria or the prevalence of helminths. Pregnant females, however, were more likely to be infected with Seoul virus than were non-pregnant females. The absence of sex differences in infection with Seoul virus and $C$. hepatica is consistent with previous studies [4, 11]. Reasons for male-biased Hymenolepis sp. infection and female-biased L. interrogans infection are unknown. Taken together, the effects of sex-related hormones, including testosterone, oestradiol, and progesterone, on the prevalence of infections in wild-caught rats may be masked by social and/or environmental factors that affect exposure $[12,13]$.
Seasonal effects were observed only for parasites (i.e. Hymenolepis sp. and spiny rat mites). Consistent with previous data, seasonal patterns in Seoul virus, $L$. interrogans, and $C$. hepatica were not observed $[4,11,14]$. Seroprevalence is not expected to show seasonal fluctuations because antibodies remain in circulation whether rats are chronically infected or have cleared the infection. Conversely, seasonal changes in the prevalence of pathogens may be pronounced because differences in the social behaviour, habitat, and environment can affect parasite populations as well as the likelihood of coming in contact with pathogens.

Pathogens that are transmitted by similar routes would be expected to infect the same individuals. Presence of antibodies against $L$. interrogans and HEV were correlated $(r=0 \cdot 36, P<0 \cdot 001)$ and both pathogens are transmitted among rat populations by ingestion of contaminated urine or faeces during social contact. Although Seoul virus also is transmitted during social contact, no correlation existed between presence of antibodies against Seoul virus and L. interrogans or HEV $(P>0 \cdot 05)$.

Seroprevalence of Seoul virus has been reported to be $\sim 50 \%$ in rats in Baltimore, Maryland $[15,16]$. Our data are consistent, as the seroprevalence was $57.7 \%$ in this survey. Rats are persistently infected for the duration of their lives and do not show signs of disease, reduced fertility, or mortality from infection [17]. Rodents release infectious virus in excrement and saliva and transmission is hypothesized to occur through inhalation of aerosolized virus in urine and faeces and passage of virus in saliva during aggressive encounters [18]. Evidence for zoonotic transmission of Seoul virus has been documented in Baltimore City populations $(0 \cdot 25 \%$ and $0.74 \%$ seroprevalences) as well as in homeless populations in Los Angeles, California $(0 \cdot 5 \%)[1,19,20]$. Disease manifestations are acute cases of haemorrhagic fever with renal syndrome (HFRS) and although the symptoms are relatively non-specific, infection is associated with hypertensive renal disease [21]. Norway rat-borne hantavirus infection occurs globally and although the mortality is low $(<5 \%)$, no effective treatment exists.

A previous study over half a century ago reported that $50.5 \%$ of wild-caught rats in Baltimore had antibodies against L. interrogans (Icterohaemorrhagiae serogroup), which is consistent with our reported $65 \cdot 3 \%$ [14]. Rats become chronically infected following contact with contaminated urine through a wound or mucous membranes. Transmission to 
humans occurs in the same manner, often in contaminated water or directly through percutaneous exposure (i.e. through cuts on the feet) in alleys, but has also been documented as being transmitted by rat bites [22, 23]. Zoonotic transmission has been demonstrated in Baltimore (16\% seroprevalence) as well as in Detroit $(31 \%)$ [22, 24]. Pathology in rats is considered to be subclinical. In humans, clinical manifestations are usually non-specific and selflimiting, but if left untreated, the disease can progress to Weil's disease, which is characterized by jaundice, acute renal failure, and possible death [22].

Antibodies against HEV have been reported in Norway rats in Baltimore $(77 \%)$, as well as in other urban centres, including Los Angeles $(73 \cdot 1 \%)[9,20]$. The current data collected in Baltimore are consistent with these findings, as $73 \%$ of the Norway rats were seropositive for HEV. Rodents and humans are primarily infected via the faecal-oral route and the self-limiting infection causes no apparent pathology in rats. Although HEV often causes subclinical disease in humans $(<1 \%$ mortality rate), it can be particularly lethal for women exposed during their third trimester of pregnancy ( $\geqslant 20 \%$ mortality) [25]. Seroprevalence in Baltimore blood donors is reported to be $21 \cdot 3 \%$, which is similar to other urban centres (i.e. Los Angeles), but few clinical cases have been diagnosed in the United States [20, 26]. The mechanism of exposure remains unknown [25].

B. elizabethae has been isolated from Norway rats in Baltimore $(10.6 \%)$ as well as from rats in other urban centres, including New Orleans $(56 \cdot 4 \%)$ [27]. In the present study, $39 \%$ of the trapped rats had detectable antibodies against $B$. elizabethae. Exposure to Bartonella sp. causes persistent circulating bacteraemia without pathology in rats. B. elizabethae is a newly emerging infection in humans and although often self-limiting, without treatment can cause potentially fatal endocarditis [27]. Antibodies to B. elizabethae have been found in inner-city injection drug users in Baltimore (33\%) [28]. The reservoir for $B$. elizabethae, as well as the mechanism of transmission, remains unknown. Rat fleas (Xenopsylla cheopis) or other ectoparasites may act as vectors for human transmission, so proximity to rats and their ectoparasites may be a risk factor for B. elizabethae infection [29].

A serological survey in Los Angeles reported higher prevalences of $R$. typhi $(25.9 \%)$ in Norway rats compared with our data (7\%) [20]. A subset of the serum samples $(n=90)$ were previously screened for $R$. typhi and the prevalence remained the same even as additional rats were included [8]. Screening for $R$. typhi rarely occurs in the absence of an outbreak, therefore little data is available for baseline seroprevalence in rats in urban centres. Transmission among rodents or from rodents to humans requires an ectoparasite vector, typically the rat or cat flea $(X$. cheopis or C. felis). Fleas do pose a potential threat, as do bloodsucking mites (i.e. the tropical rat mite $O$. bacoti). Two tropical rat mites were collected from seropositive rats and were tested for the presence of $R$. typhi DNA, but were negative. $R$. typhi is the aetiological agent of murine typhus, an often self-limiting febrile illness which can cause complications in immunocompromised populations ( $1 \%$ mortality rate).

The prevalence of Hymenolepis sp. in urban centres has not been previously reported and we reported a prevalence of $34.0 \%$ in Baltimore. Both dwarf tapeworms (H. nana) and rat tapeworms $(H$. diminuta) are transmitted by insect vectors; however, infectious ova from $H$. nana can also be spread by the faecal-oral route. Humans and other animals become infected when they eat material contaminated by infected insects or faeces. Both rat and human infections are usually subclinical, but symptoms such as gastrointestinal system discomfort and diarrhoea, can ensue during heavy infections.

A previous study reported an $87.4 \%$ prevalence of Calodium hepatica in Norway rats in residental areas of Baltimore, which is consistent with the $87.9 \%$ prevalence reported in this survey [11]. C. hepatica is primarily transmitted by predation and ingestion of ova in the host liver. Release of ova that embryonate in the surrounding environment can pose a threat if ingested. Human cases of capillariasis are rare, but can result in liver damage and fatality [11].

Previous data revealed that LCMV is detected in $4.7 \%$ of inner-city Baltimore residents and has been found in house mice (Mus musculus) in Baltimore $(9 \cdot 0 \%)[1,30]$. Natural LCMV infection has not been reported in Norway rats. No rats tested positive for LCMV; therefore we conclude that rats do not act as a vector for LCMV infection in humans.

In summary, this survey of zoonotic pathogens provides important background seroprevalences and prevalences in the absence of outbreaks. Interpretation of serological analyses has some limitations, including potential cross-reactivity, sensitivity, and specificity limitations. The mode of transmission, prevalence in rodent populations, and duration of infection influence the risk of zoonotic transmission 
to humans. The presence of antibody does not necessarily indicate an ongoing infection; therefore, the duration of infection (i.e. acute $v s$. chronic) is an important factor in considering risk of transmission. For example, a pathogen that is aerosolized, is highly prevalent in rat populations, and chronically infects rats would pose a high risk of transmission to humans. Of the pathogens evaluated, the highest risk to humans is probably transmission of L. interrogans, followed by $H$. nana and Seoul virus. Because these pathogens are prevalent in rat populations, chronically infect rats, and are shed in excrement and saliva, potential for human contact is high in urban environments. Conversely, pathogens that require an ectoparasite vector (i.e. $R$. typhi, and $H$. diminuta) and are found at a low prevalence are not as likely to infect humans in an urban setting. The most prevalent ectoparasite was the spiny rat mite, which has not been reported to harbour any zoonotic pathogens. The less prevalent tropical rat mite and cat flea, however, have been implicated in zoonoses, including murine typhus, rickettsialpox, plague, and cat scratch fever. As fleas and mesostigmatid mites are typically found on the host only during feeding, the actual prevalence in this population may be higher than we report here. The risk of transmission of HEV or B. elizabethae from rats to humans remains unknown, as unexplained exposure in human populations occurs (15-20\% seroprevalences). C. hepatica and LCMV infections do not appear to be transmitted from rats to humans. Estimated morbidity and mortality due to rodent-borne zoonoses in industrialized nations has not been evaluated. Due to the non-specific symptoms caused by these rodent-borne zoonotic pathogens, transmission to human populations probably goes underdiagnosed due to lack of clinical suspicion.

\section{ACKNOWLEDGEMENTS}

We thank Darren Kaw for help with trapping the rats during summer 2005 and Andrew Glenn and Bruce Baldwin for with help in identification of helminth ova, Dr A. D. Loftis for her help with rickettsial serology, and Ronald Engle (NIAID) for performing the anti-HEV tests. Financial support was provided by NIH grant R01 A1054995 (S. L. K.) and NSF grant EF0525751 (G.E.G.).

\section{DECLARATION OF INTEREST}

None.

\section{REFERENCES}

1. Childs JE, et al. Human-rodent contact and infection with lymphocytic choriomeningitis and Seoul viruses in an inner-city population. American Journal of Tropical Medicine and Hygiene 1991 ; 44: 117-121.

2. Webster JP, Macdonald DW. Parasites of wild brown rats (Rattus norvegicus) on UK farms. Parasitology 1995; 111 : 247-255.

3. Glass GE, et al. Association of intraspecific wounding with hantaviral infection in wild rats (Rattus norvegicus). Epidemiology and Infection 1988; 101: 459-472.

4. Klein SL, et al. Environmental and physiological factors associated with Seoul virus infection among urban populations of Norway rats. Journal of Mammalogy $2002 ; 83: 478-488$.

5. Engle RE, et al. Hepatitis E virus (HEV) capsid antigens derived from viruses of human and swine origin are equally efficient for detecting anti-HEV by enzyme immunoassay. Journal of Clinical Microbiology 2002; 40: 4576-4580.

6. Robinson RA, et al. Structural characterization of recombinant hepatitis $\mathrm{E}$ virus ORF2 proteins in baculovirus-infected insect cells. Protein Expression and Purification 1998; 12: 75-84.

7. Vanasco NB, et al. Development and validation of an ELISA for the detection of leptospire-specific antibodies in rodents. Veterinary Microbiology 2001; 82: 321-330.

8. Reeves WK, et al. Serologic evidence for Rickettsia typhi and an ehrlichial agent in Norway rats from Baltimore, Maryland, USA. Vector-Borne and Zoonotic Diseases 2006; 6: 244-247.

9. Kabrane-Lazizi Y, et al. Evidence for widespread infection of wild rats with hepatitis E virus in the United States. American Journal of Tropical Medicine and Hygiene 1999; 61: 331-335.

10. Vanasco NB, et al. Associations between leptospiral infection and seropositivity in rodents and environmental characteristics in Argentina. Preventative Veterinary Medicine 2003; 60: 227-235.

11. Childs JE, Glass GE, Korch GW. The comparative epizootiology of Capillaria hepatica (Nematoda) in urban rodents from different habitats of Baltimore, Maryland. Canadian Journal of Zoology 1988; 66: 2769-2775.

12. Klein SL, et al. Neonatal sex steroids affect responses to Seoul virus infection in male but not female Norway rats. Brain, Behavior, and Immunity 2002; 16: 736-746.

13. Klein SL, Zink MC, Glass GE. Seoul virus infection increases aggressive behaviour in male Norway rats. Animal Behaviour 2004; 67: 421-429.

14. Li HY, Davis DE. The prevalence of carriers of Leptospira and Salmonella in Norway rats of Baltimore. American Journal of Hygiene 1952; 56: 90-100.

15. Easterbrook JD, et al. Norway rat population in Baltimore, Maryland, 2004. Vector-Borne and Zoonotic Diseases 2005; 5: 296-299. 
16. Hinson ER, et al. Wounding: the primary mode of Seoul virus transmission among male Norway rats. American Journal of Tropical Medicine and Hygiene 2004; 70: 310-317.

17. Hjelle B, Yates T. Modeling hantavirus maintenance and transmission in rodent communities. In: Hantaviruses, 2001, pp. 77-90.

18. Kawamata $\mathbf{J}$, et al. Control of laboratory acquired hemorrhagic fever with renal syndrome (HFRS) in Japan. Laboratory Animal Science 1987; 37: 431-436.

19. Diglisic G, et al. Seroprevalence study of hantavirus infection in the community based population. Maryland Medical Journal 1999; 48: 303-306.

20. Smith HM, et al. Prevalence study of antibody to ratborne pathogens and other agents among patients using a free clinic in downtown Los Angeles. Journal of Infectious Diseases 2002; 186: 1673-1676.

21. Glass GE, et al. Domestic cases of hemorrhagic fever with renal syndrome in the United States. Nephron 1994; 68: 48-51.

22. Vinetz JM, et al. Sporadic urban leptospirosis. Annals of Internal Medicine 1996; 125: 794-798.

23. Gollop JH, et al. Rat-bite leptospirosis. Western Journal of Medicine 1993; 159: 76-77.
24. Demers RY, et al. Exposure to Leptospira icterohaemorrhagiae in inner-city and suburban children: a serologic comparison. Journal of Family Practice 1983; 17: 1007-1111.

25. Emerson SU, Purcell RH. Hepatitis E virus. Reviews in Medical Virology 2003; 13: 145-154.

26. Thomas DL, et al. Seroreactivity to hepatitis E virus in areas where the disease is not endemic. Journal of Clinical Microbiology 1997; 35: 1244-1247.

27. Ellis BA, et al. Rats of the genus Rattus are reservoir hosts for pathogenic Bartonella species: an Old World origin for a New World disease? Journal of Infectious Diseases 1999; 180: 220-224.

28. Comer JA, et al. Antibodies to Bartonella species in inner-city intravenous drug users in Baltimore, MD. Archives of Internal Medicine 1996; 156: 2491-2495.

29. Kosoy MY, et al. Distribution, diversity, and host specificity of Bartonella in rodents from the Southeastern United States. American Journal of Tropical Medicine and Hygiene 1997; 57: 578-588.

30. Childs JE, et al. Lymphocytic choriomeningitis virus infection and house mouse (Mus musculus) distribution in urban Baltimore. American Journal of Tropical Medicine and Hygiene 1992; 47: 27-34. 